\title{
Nonlocal boundary value problems for fractional differential inclusions with Erdélyi-Kober fractional integral boundary conditions
}

\author{
Sotiris K. Ntouyas ${ }^{\mathrm{a}, \mathrm{b}}$, Jessada Tariboon ${ }^{\mathrm{c}, *}$ \\ a Department of Mathematics, University of Ioannina, 45110 Ioannina, Greece \\ b Nonlinear Analysis and Applied Mathematics (NAAM)-Research Group, Department of Mathematics, \\ Faculty of Science, King Abdulaziz University, P.O. Box 80203, Jeddah 21589, Saudi Arabia \\ ${ }^{c}$ Nonlinear Dynamic Analysis Research Centre, Department of Mathematics, Faculty of Applied Science, \\ King Mongkut's University of Technology North Bangkok, Bangkok 10800 Thailand
}

*Corresponding author, e-mail: jessada.t@sci.kmutnb.ac.th

Received 2 Mar 2016

Accepted 16 Mar 2017

\begin{abstract}
We study a new class of boundary value problems consisting of a fractional differential inclusion of Riemann-Liouville type and Erdélyi-Kober fractional integral conditions. Some new existence results for convex as well as nonconvex multivalued maps are obtained by using standard fixed-point theorems. Some illustrative examples are also presented.
\end{abstract}

KEYWORDS: Riemann-Liouville fractional derivative, fixed point theorem

MSC2010: 34A60 34A08 34A12 34B15

\section{INTRODUCTION}

There is considerable interest in fractional differential equations due to their numerous applications, e.g., modelling of viscoelastic behaviour ${ }^{1}$, anomalous diffusion ${ }^{2}$, cellular neural networks ${ }^{3}$, and long-time memory in financial time series via fractional Langevin equations ${ }^{4}$. For further details and examples see Refs. 5-10. For some recent development of the topic see Refs. 11-14 and the references cited therein. Most of the work on the topic involves the Riemann-Liouville or Caputo type fractional derivative. The so-called ErdélyiKober fractional derivative, which is a generalization of the Riemann-Liouville fractional derivative, is often used too. An Erdélyi-Kober operator is a fractional integration operation ${ }^{15}$. These operators have been used by many authors, in particular, to obtain solutions of the single, dual, and triple integral equations possessing special functions as their kernels ${ }^{6,15-18}$.

We consider the boundary value problem

$$
\left.\begin{array}{l}
D^{q} x(t) \in F(t, x(t)), 0<t<T, 1<q<2, \\
x(0)=0, \quad \alpha x(T)=\sum_{i=1}^{m} \beta_{i} I_{\eta_{i}}^{\gamma_{i}, \delta_{i}} x\left(\xi_{i}\right),
\end{array}\right\}
$$

where $D^{q}$ is the standard Riemann-Liouville fractional derivative of order $q, I_{\eta_{i}}^{\gamma_{i}, \delta_{i}}$ is the ErdélyiKober fractional integral of order $\delta_{i}>0$ with $\eta_{i}>0$ and $\gamma_{i} \in \mathbb{R}, i=1,2, \ldots, m, F:[0, T] \times \mathbb{R} \rightarrow \mathscr{P}(\mathbb{R})$ is a multivalued map, $\mathscr{P}(\mathbb{R})$ is the family of all nonempty subsets of $\mathbb{R}$, and $\alpha, \beta_{i} \in \mathbb{R}, \xi_{i} \in(0, T)$, $i=1,2, \ldots, m$ are given constants.

The present paper is motivated by Ref. 19 where the problem (1) was studied for single-valued maps, i.e., $F=\{f\}$. Here we cover the multivalued case. We establish some existence results for the problem (1) when the right-hand side has convex as well as nonconvex values. In the case of convex values (upper semicontinuous case) we use the nonlinear alternative of Leray-Schauder type. When the righthand side is not necessarily convex valued (lower semicontinuous case) we combine the nonlinear alternative of Leray-Schauder type for single-valued maps with a selection theorem for lower semicontinuous multivalued maps with nonempty closed and decomposable values. Finally, we prove the existence of solutions for the problem (1) with a not necessarily nonconvex-valued right-hand side by applying a fixed-point theorem for contractive multivalued maps Although the methods used are well known, their exposition in the framework of 
problem (1) is new.

\section{PRELIMINARIES}

\section{Basic material for fractional calculus}

In this section, we introduce some notation and definitions of fractional calculus and present results needed later.

The Riemann-Liouville fractional derivative of order $q>0$ of a continuous function $f:(0, \infty) \rightarrow \mathbb{R}$ is defined by

$$
D^{q} f(t)=\frac{1}{\Gamma(n-q)} \frac{\mathrm{d}^{n}}{\mathrm{~d} t^{n}} \int_{0}^{t}(t-s)^{n-q-1} f(s) \mathrm{d} s,
$$

where $\Gamma(x)$ is the Gamma function of $x, n$ is a positive integer, and $n-1<q<n$.

The Riemann-Liouville fractional integral of order $q>0$ of a continuous function $f:(0, \infty) \rightarrow \mathbb{R}$ is defined by

$$
J^{q} f(t)=\frac{1}{\Gamma(q)} \int_{0}^{t}(t-s)^{q-1} f(s) \mathrm{d} s,
$$

provided the integral exists.

The Erdélyi-Kober fractional integral of order $\delta>$ 0 with $\eta>0$ and $\gamma \in \mathbb{R}$ of a continuous function $f:(0, \infty) \rightarrow \mathbb{R}$ is defined by

$$
I_{\eta}^{\gamma, \delta} f(t)=\frac{\eta t^{-\eta(\delta+\gamma)}}{\Gamma(\delta)} \int_{0}^{t} \frac{s^{\eta \gamma+\eta-1} f(s)}{\left(t^{\eta}-s^{\eta}\right)^{1-\delta}} \mathrm{d} s
$$

provided the right-hand side is pointwise defined on $\mathbb{R}_{+}$. For $\eta=1$ this operator reduces to the Kober operator $^{15}$

$$
I_{\gamma}^{\delta} f(t)=\frac{t^{-(\delta+\gamma)}}{\Gamma(\delta)} \int_{0}^{t} \frac{s^{\gamma} f(s)}{(t-s)^{1-\delta}} \mathrm{d} s, \quad \gamma, \delta>0 .
$$

For $\gamma=0$, the Kober operator reduces to the Riemann-Liouville fractional integral with a power weight:

$$
I_{0}^{\delta} f(t)=\frac{t^{-\delta}}{\Gamma(\delta)} \int_{0}^{t} \frac{f(s)}{(t-s)^{1-\delta}} \mathrm{d} s, \quad \delta>0 .
$$

Lemma 1 (Ref. 6) Let $q>0$ and $y \in C(0, T) \cap$ $L(0, T)$. Then the fractional differential equation $D^{q} y(t)=0$ has a unique solution

$$
y(t)=c_{1} t^{q-1}+c_{2} t^{q-2}+\cdots+c_{n} t^{q-n},
$$

where $c_{i} \in \mathbb{R}, i=1,2, \ldots, n$ and $n-1<q<n$.

Lemma 2 (Ref. 6) Let $q>0$. Then for $y \in C(0, T) \cap$ $L(0, T)$,

$$
J^{q} D^{q} y(t)=y(t)+c_{1} t^{q-1}+c_{2} t^{q-2}+\cdots+c_{n} t^{q-n},
$$

where $c_{i} \in \mathbb{R}, i=1,2, \ldots, n$ and $n-1<q<n$.

\section{Some auxiliary lemmas}

Lemma 3 (Ref. 19) Let $\delta, \eta>0$ and $\gamma, q \in \mathbb{R}$. Then

$$
I_{\eta}^{\gamma, \delta} t^{q}=\frac{t^{q} \Gamma(\gamma+q / \eta+1)}{\Gamma(\gamma+q / \eta+\delta+1)} .
$$

Lemma 4 (Ref. 19) Let $1<q<2, \delta_{i}, \eta_{i}>0$, $\alpha, \gamma_{i}, \beta_{i} \in \mathbb{R}, \xi_{i} \in(0, T), i=1,2, \ldots, m$ and $h \in$ $C([0, T], \mathbb{R})$. Then the linear Riemann-Liouville fractional differential equation subject to the ErdélyiKober fractional integral boundary conditions

$$
\left.\begin{array}{l}
D^{q} x(t)=h(t), \quad t \in(0, T), \\
x(0)=0, \quad \alpha x(T)=\sum_{i=1}^{m} \beta_{i} I_{\eta_{i}}^{\gamma_{i}, \delta_{i}} x\left(\xi_{i}\right),
\end{array}\right\}
$$

is equivalent to the integral equation

$$
\begin{aligned}
x(t)=J^{q} h(t)-\frac{t^{q-1}}{\Lambda} & \left(\alpha J^{q} h(T)\right. \\
& \left.-\sum_{i=1}^{m} \beta_{i} I_{\eta_{i}}^{\gamma_{i}, \delta_{i}} J^{q} h\left(\xi_{i}\right)\right),
\end{aligned}
$$

where

$$
\begin{aligned}
\Lambda:= & \alpha T^{q-1} \\
& -\sum_{i=1}^{m} \frac{\beta_{i} \xi_{i}^{q-1} \Gamma\left(\gamma_{i}+(q-1) / \eta_{i}+1\right)}{\Gamma\left(\gamma_{i}+(q-1) / \eta_{i}+\delta_{i}+1\right)} \neq 0 .
\end{aligned}
$$

\section{Basic material for multivalued maps}

Here we outline some basic concepts of multivalued analysis $^{20,21}$. Let $C([0, T], \mathbb{R})$ denote the Banach space of all continuous functions from $[0, T]$ into $\mathbb{R}$ with the norm $\|x\|=\sup \{|x(t)|, t \in[0, T]\}$. Also by $L^{1}([0, T], \mathbb{R})$ we denote the space of functions $x:[0, T] \rightarrow \mathbb{R}$ such that $\|x\|_{L^{1}}=\int_{0}^{T}|x(t)| \mathrm{d} t$.

For a normed space $(X,\|\cdot\|)$, let

$$
\begin{aligned}
& \mathscr{P}_{\mathrm{cl}}(X)=\{Y \in \mathscr{P}(X): Y \text { is closed }\}, \\
& \mathscr{P}_{\mathrm{b}}(X)=\{Y \in \mathscr{P}(X): Y \text { is bounded }\},
\end{aligned}
$$

$\mathscr{P}_{\mathrm{cl}, \mathrm{b}}(X)=\{Y \in \mathscr{P}(X): Y$ is closed and bounded $\}$, $\mathscr{P}_{\mathrm{cp}}(X)=\{Y \in \mathscr{P}(X): Y$ is compact $\}$, $\mathscr{P}_{\mathrm{cp}, \mathrm{c}}(X)=\{Y \in \mathscr{P}(X): Y$ is compact and convex $\}$.

A multivalued map $G: X \rightarrow \mathscr{P}(X)$ is convex (closed) valued if $G(x)$ is convex (closed) for all $x \in$ $X$. $G$ is bounded on bounded sets if $G(Y)=$ $\cup_{x \in Y} G(x)$ is bounded in $X$ for all $Y \in \mathscr{P}_{\mathrm{b}}(X)$ (i.e., $\left.\sup _{x \in Y}\{\sup \{|y|: y \in G(x)\}\}<\infty\right) . \quad G$ is called upper semicontinuous (u.s.c.) on $X$ if for each $x_{0} \in X$ 
the set $G\left(x_{0}\right)$ is a nonempty closed subset of $X$ and if for each open set $N$ of $X$ containing $G\left(x_{0}\right)$ there exists an open neighbourhood $\mathscr{N}_{0}$ of $x_{0}$ such that $G\left(\mathscr{N}_{0}\right) \subseteq N . \quad G$ is lower semicontinuous if the set $\{y \in X: G(y) \cap Y \neq \varnothing\}$ is open for any open set $Y$ in $X . G$ is said to be completely continuous if $G(B)$ is relatively compact for every $B \in \mathscr{P}_{\mathrm{b}}(X)$; if the multivalued map $G$ is completely continuous with nonempty compact values then $G$ is u.s.c. if and only if $G$ has a closed graph, i.e., $x_{n} \rightarrow x_{*}$, $y_{n} \rightarrow y_{*}, y_{n} \in G\left(x_{n}\right)$ imply $y_{*} \in G\left(x_{*}\right)$. $G$ is said to be measurable if for every $y \in X$, the function

$$
t \mapsto d(y, G(t))=\inf \{|y-z|: z \in G(t)\}
$$

is measurable. $G$ has a fixed point if there is $x \in$ $X$ such that $x \in G(x)$. The fixed point set of the multivalued operator $G$ will be denoted by Fix $G$.

\section{EXISTENCE RESULTS}

\section{The Carathéodory case}

In this subsection we consider the case when $F$ has convex values and prove an existence result based on a nonlinear alternative of Leray-Schauder type, assuming that $F$ is Carathéodory.

A multivalued map $F:[0, T] \times \mathbb{R} \rightarrow \mathscr{P}(\mathbb{R})$ is said to be Carathéodory if (i) $t \mapsto F(t, x)$ is measurable for each $x \in \mathbb{R}$ and (ii) $x \mapsto F(t, x)$ is u.s.c. for almost all $t \in[0, T]$. A Carathéodory function $F$ is called $L^{1}$-Carathéodory if for each $\rho>0$, there exists $\varphi_{\rho} \in L^{1}\left([0, T], \mathbb{R}^{+}\right)$such that

$$
\|F(t, x)\|=\sup \{|v|: v \in F(t, x)\} \leqslant \varphi_{\rho}(t)
$$

for all $\|x\| \leqslant \rho$ and for almost all $t \in[0, T]$.

For each $y \in C([0, T], \mathbb{R})$, define the set of selections of $F$ by

$$
\begin{array}{r}
S_{F, y}:=\left\{v \in L^{1}([0, T], \mathbb{R}): v(t) \in F(t, y(t))\right. \\
\text { on }[0, T]\} .
\end{array}
$$

We define the graph of $G$ to be the set $\operatorname{Gr}(G)=$ $\{(x, y) \in X \times Y, y \in G(x)\}$ and recall a result for closed graphs and upper semicontinuity.

Lemma 5 (Ref. 20) If $G: X \rightarrow \mathscr{P}_{\mathrm{cl}}(Y)$ is u.s.c. then $\operatorname{Gr}(G)$ is a closed subset of $X \times Y$, i.e., for every sequence $\left\{x_{n}\right\}_{n \in \mathbb{N}} \subset X$ and $\left\{y_{n}\right\}_{n \in \mathbb{N}} \subset Y$, if when $n \rightarrow \infty, x_{n} \rightarrow x_{*}, y_{n} \rightarrow y_{*}$ and $y_{n} \in G\left(x_{n}\right)$, then $y_{*} \in G\left(x_{*}\right)$. Conversely, if $G$ is completely continuous and has a closed graph, then it is u.s.c.

Lemma 6 (Ref. 22) Let $X$ be a Banach space. Let $F$ : $J \times \mathbb{R} \rightarrow \mathscr{P}_{\mathrm{cp}, \mathrm{c}}(X)$ be an $L^{1}$-Carathéodory multivalued map and let $\Theta$ be a linear continuous mapping from $L^{1}(J, X)$ to $C(J, X)$. Then the operator

$$
\begin{gathered}
\Theta \circ S_{F}: C(J, X) \rightarrow \mathscr{P}_{\mathrm{cp}, \mathrm{c}}(C(J, X)), \\
x \mapsto\left(\Theta \circ S_{F}\right)(x)=\Theta\left(S_{F, x}\right)
\end{gathered}
$$

is a closed graph operator in $C(J, X) \times C(J, X)$.

We recall the well-known nonlinear alternative of Leray-Schauder type for multivalued (Kakutani) maps.

Lemma 7 (Ref. 23) Let $E$ be a Banach space, $C$ a closed convex subset of $E, U$ an open subset of $C$ and $0 \in U$. Suppose that $F: \bar{U} \rightarrow \mathscr{P}_{\mathrm{cp}, \mathrm{c}}(C)$ is a u.s.c. compact map. Then either (i) $F$ has a fixed point in $\bar{U}$, or (ii) there is $a u \in \partial U$ and $\lambda \in(0,1)$ with $u \in \lambda F(u)$.

Throughout this paper, for convenience, we use the following expressions

$$
J^{q} f(s)(z)=\frac{1}{\Gamma(q)} \int_{0}^{z}(z-s)^{q-1} f(s) \mathrm{d} s, \quad z \in\{t, T\},
$$

for $t \in[0, T]$ and

$$
\begin{aligned}
& I_{\eta_{i}}^{\gamma_{i}, \delta_{i}} J^{q} f(s)\left(\xi_{i}\right)= \frac{\eta_{i} \xi_{i}^{-\eta_{i}\left(\delta_{i}+\gamma_{i}\right)}}{\Gamma(q) \Gamma\left(\delta_{i}\right)} \\
& \times \int_{0}^{\xi_{i}} \int_{0}^{r} \frac{r^{\eta_{i} \gamma_{i}+\eta_{i}-1}(r-s)^{q-1}}{\left(\xi_{i}^{\eta_{i}}-r \eta_{i}\right)^{1-\delta_{i}}} f(s) \mathrm{d} s \mathrm{~d} r,
\end{aligned}
$$

where $\xi_{i} \in(0, T)$ for $i=1,2, \ldots, m$.

Theorem 1 Assume that

$\left(\mathrm{H}_{1}\right) F:[0, T] \times \mathbb{R} \rightarrow \mathscr{P}_{\mathrm{cp}, \mathrm{c}}(\mathbb{R})$ is $L^{1}$-Carathéodory;

$\left(\mathrm{H}_{2}\right)$ there exists a continuous nondecreasing function $\psi:[0, \infty) \rightarrow(0, \infty)$ and a function $p \in$ $C\left([0, T], \mathbb{R}^{+}\right)$such that

$$
\begin{aligned}
\|F(t, x)\|_{\mathscr{P}} & :=\sup \{|y|: y \in F(t, x)\} \\
& \leqslant p(t) \psi(\|x\|)
\end{aligned}
$$

for each $(t, x) \in[0, T] \times \mathbb{R}$;

$\left(\mathrm{H}_{3}\right)$ there exists a constant $M>0$ such that $M / \psi(M)\|p\| \Psi>1$, where

$$
\begin{array}{r}
\Psi=\frac{T^{q}}{\Gamma(q+1)}+\frac{|\alpha| T^{2 q-1}}{|\Lambda| \Gamma(q+1)}+\frac{T^{q-1}}{|\Lambda| \Gamma(q+1)} \\
\times \sum_{i=1}^{m} \frac{\left|\beta_{i}\right| \xi_{i}^{q} \Gamma\left(\gamma_{i}+q / \eta_{i}+1\right)}{\Gamma\left(\gamma_{i}+q / \eta_{i}+\delta_{i}+1\right)} .
\end{array}
$$

Then the boundary value problem (1) has at least one solution on $[0, T]$. 
Proof: Define the operator $\mathscr{F}: C([0, T], \mathbb{R}) \rightarrow$ $\mathscr{P}(C([0, T], \mathbb{R}))$ by

$$
\mathscr{F}(x)=\{h \in C([0, T], \mathbb{R}): h(t)=v(t)\}
$$

where

$$
\begin{aligned}
v(t)=J^{q} v(s)(t)-\frac{t^{q-1}}{\Lambda} & \left(\alpha J^{q} v(s)(T)\right. \\
& \left.-\sum_{i=1}^{m} \beta_{i} I_{\eta_{i}}^{\gamma_{i}, \delta_{i}} J^{q} v(s)\left(\xi_{i}\right)\right)
\end{aligned}
$$

for $v \in S_{F, x}$. It is obvious that the fixed points of $\mathscr{F}$ are solutions of the boundary value problem (1).

We now show that $\mathscr{F}$ satisfies the assumptions of Leray-Schauder Nonlinear alternative (Lemma 7). The proof consists of several steps.

Step 1: $\mathscr{F}(x)$ is convex for each $x \in$ $C([0, T], \mathbb{R})$. This step is obvious since $S_{F, x}$ is convex ( $F$ has convex values).

Step 2: $\mathscr{F}$ maps bounded sets (balls) into bounded sets in $C([0, T], \mathbb{R})$. For a positive number $\rho$, let $B_{\rho}=\{x \in C([0, T], \mathbb{R}):\|x\| \leqslant \rho\}$ be a bounded ball in $C([0, T], \mathbb{R})$. Then, for each $h \in$ $\mathscr{F}(x), x \in B_{\rho}$, there exists $v \in S_{F, x}$ such that

$$
\begin{aligned}
h(t)=J^{q} v(s)(t)-\frac{t^{q-1}}{\Lambda} & \left(\alpha J^{q} v(s)(T)\right. \\
& \left.-\sum_{i=1}^{m} \beta_{i} I_{\eta_{i}}^{\gamma_{i}, \delta_{i}} J^{q} v(s)\left(\xi_{i}\right)\right) .
\end{aligned}
$$

Then we have

$$
\begin{aligned}
|h(x)| \leqslant J^{q}|v(s)|(T)+\frac{|\alpha| T^{q-1}}{|\Lambda|} J^{q}|v(s)|(T) & \\
+ & \frac{T^{q-1}}{|\Lambda|} \sum_{i=1}^{m}\left|\beta_{i}\right| I_{\eta_{i}}^{\gamma_{i}, \delta_{i}} J^{q}|v(s)|\left(\xi_{i}\right) \\
\leqslant & \psi(\|x\|)\left(J^{q} p(s)(T)+\frac{|\alpha| T^{q-1}}{|\Lambda|} J^{q} p(s)(T)\right. \\
& \left.+\frac{T^{q-1}}{|\Lambda|} \sum_{i=1}^{m}\left|\beta_{i}\right| I_{\eta_{i}}^{\gamma_{i}, \delta_{i}} J^{q} p(s)\left(\xi_{i}\right)\right) \\
\leqslant & \psi(\|x\|)\|p\|\left(\frac{T^{q}}{\Gamma(q+1)}+\frac{|\alpha| T^{2 q-1}}{|\Lambda| \Gamma(q+1)}\right. \\
& \left.+\frac{T^{q-1}}{|\Lambda| \Gamma(q+1)} \sum_{i=1}^{m} \frac{\left|\beta_{i}\right| \xi_{i}^{q} \Gamma\left(\gamma_{i}+q / \eta_{i}+1\right)}{\Gamma\left(\gamma_{i}+q / \eta_{i}+\delta_{i}+1\right)}\right)
\end{aligned}
$$

and consequently, $\|h\| \leqslant \psi(r)\|p\| \Psi$.

Step 3: $\mathscr{F}$ maps bounded sets into equicontinuous sets of $C([0, T], \mathbb{R})$. Let $\tau_{1}, \tau_{2} \in[0, T]$ with $\tau_{1}<\tau_{2}$ and $x \in B_{\rho}$. For each $h \in \mathscr{F}(x)$ we obtain

$$
\begin{array}{rl}
\mid h\left(\tau_{2}\right) & -h\left(\tau_{1}\right) \mid \\
\leqslant \mid J^{q} & v(s)\left(\tau_{2}\right)-J^{q} v(s)\left(\tau_{1}\right) \mid \\
& +\frac{|\alpha|\left|\tau_{2}^{q-1}-\tau_{1}^{q-1}\right|}{|\Lambda|} J^{q}|v(s)|(T) \\
& +\frac{\left|\tau_{2}^{q-1}-\tau_{1}^{q-1}\right|}{|\Lambda|} \sum_{i=1}^{m}\left|\beta_{i}\right| I_{\eta_{i}}^{\gamma_{i}, \delta_{i}} J^{q}|v(s)|\left(\xi_{i}\right) \\
\leqslant & \frac{\psi(r)}{\Gamma(q)} \mid \int_{0}^{\tau_{1}}\left[\left(\tau_{2}-s\right)^{q-1}-\left(\tau_{1}-s\right)^{q-1}\right] p(s) \mathrm{d} s \\
& +\int_{\tau_{1}}^{\tau_{2}}\left(\tau_{2}-s\right)^{q-1} p(s) \mathrm{d} s \mid \\
+ & \frac{\left|\tau_{2}^{q-1}-\tau_{1}^{q-1}\right| \psi(r)}{|\Lambda|}\left(|\alpha| J^{q} p(s)(T)\right. \\
& \left.+\sum_{i=1}^{m}\left|\beta_{i}\right| I_{\eta_{i}}^{\gamma_{i}, \delta_{i}} J^{q} p(s)\left(\xi_{i}\right)\right) .
\end{array}
$$

Clearly, the right-hand side of the above inequality tends to zero independently of $x \in B_{\rho}$ as $\tau_{2}-\tau_{1} \rightarrow$ 0 . As $\mathscr{F}$ satisfies the above three assumptions, it follows by the Ascoli-Arzelá theorem that $\mathscr{F}$ : $C([0, T], \mathbb{R}) \rightarrow \mathscr{P}(C([0, T], \mathbb{R}))$ is completely continuous. Since $\mathscr{F}$ is completely continuous, in order to prove that it is u.s.c. it is enough to prove that it has a closed graph.

Step 4: $\mathscr{F}$ has a closed graph. Let $x_{n} \rightarrow x_{*}, h_{n} \in$ $\mathscr{F}\left(x_{n}\right)$ and $h_{n} \rightarrow h_{*}$. Then we need to show that $h_{*} \in \mathscr{F}\left(x_{*}\right)$. Associated with $h_{n} \in \mathscr{F}\left(x_{n}\right)$ there exists $v_{n} \in S_{F, x_{n}}$ such that for each $t \in[0, T]$,

$$
\begin{aligned}
h_{n}(t)=J^{q} v_{n}(s)(t)- & \frac{t^{q-1}}{\Lambda}\left(\alpha J^{q} v_{n}(s)(T)\right. \\
& \left.-\sum_{i=1}^{m} \beta_{i} I_{\eta_{i}}^{\gamma_{i}, \delta_{i}} J^{q} v_{n}(s)\left(\xi_{i}\right)\right) .
\end{aligned}
$$

Thus it suffices to show that there exists $v_{*} \in S_{F, x_{*}}$ such that for each $t \in[0, T]$,

$$
\begin{aligned}
h_{*}(t)=J^{q} v_{*}(s)(t)- & \frac{t^{q-1}}{\Lambda}\left(\alpha J^{q} v_{*}(s)(T)\right. \\
& \left.-\sum_{i=1}^{m} \beta_{i} I_{\eta_{i}}^{\gamma_{i}, \delta_{i}} J^{q} v_{*}(s)\left(\xi_{i}\right)\right) .
\end{aligned}
$$

Consider the linear operator $\Theta: L^{1}([0, T], \mathbb{R}) \rightarrow$ 
$C([0, T], \mathbb{R})$ given by

$$
\begin{array}{r}
f \mapsto \Theta(v)(t)=J^{q} v(s)(t)-\frac{t^{q-1}}{\Lambda}\left(\alpha J^{q} v(s)(T)\right. \\
\left.-\sum_{i=1}^{m} \beta_{i} I_{\eta_{i}}^{\gamma_{i}} \delta_{i} J^{q} v(s)\left(\xi_{i}\right)\right) .
\end{array}
$$

Observe that

$$
\left\|h_{n}(t)-h_{*}(t)\right\| \rightarrow 0, \quad \text { as } n \rightarrow \infty .
$$

Thus it follows by Lemma 6 that $\Theta \circ S_{F}$ is a closed graph operator. Further, we have $h_{n}(t) \in \Theta\left(S_{F, x_{n}}\right)$. Since $x_{n} \rightarrow x_{*}$, we have

$$
\begin{aligned}
h_{*}(t)=J^{q} v_{*}(s)(t)- & \frac{t^{q-1}}{\Lambda}\left(\alpha J^{q} v_{*}(s)(T)\right. \\
& \left.-\sum_{i=1}^{m} \beta_{i} I_{\eta_{i}}^{\gamma_{i}, \delta_{i}} J^{q} v_{*}(s)\left(\xi_{i}\right)\right),
\end{aligned}
$$

for some $v_{*} \in S_{F, x_{*}}$.

Step 5: there exists an open set $U \subseteq C([0, T], \mathbb{R})$ with $x \notin \lambda \mathscr{F}(x)$ for any $\lambda \in(0,1)$ and all $x \in \partial U$. Let $\lambda \in(0,1)$ and $x \in \lambda \mathscr{F}(x)$. Then there exists $v \in$ $L^{1}([0, T], \mathbb{R})$ with $v \in S_{F, x}$ such that, for $t \in[0, T]$,

$$
\begin{aligned}
x(t)=\lambda J^{q} v(s)(t)- & \lambda \frac{t^{q-1}}{\Lambda}\left(\alpha J^{q} v(s)(T)\right. \\
& \left.-\sum_{i=1}^{m} \beta_{i} I_{\eta_{i}}^{\gamma_{i}, \delta_{i}} J^{q} v(s)\left(\xi_{i}\right)\right) .
\end{aligned}
$$

Using the computations of the second step above,

$$
\begin{aligned}
\|x\| & \leqslant \psi(\|x\|)\|p\|\left\{\frac{T^{q}}{\Gamma(q+1)}+\frac{|\alpha| T^{2 q-1}}{|\Lambda| \Gamma(q+1)}\right. \\
& \left.+\frac{T^{q-1}}{|\Lambda| \Gamma(q+1)} \sum_{i=1}^{m} \frac{\left|\beta_{i}\right| \xi_{i}^{q} \Gamma\left(\gamma_{i}+q / \eta_{i}+1\right)}{\Gamma\left(\gamma_{i}+q / \eta_{i}+\delta_{i}+1\right)}\right\} \\
& =\psi(\|x\|)\|p\| \Psi,
\end{aligned}
$$

which implies that

$$
\frac{\|x\|}{\psi(\|x\|)\|p\| \Psi} \leqslant 1 .
$$

In view of $\left(\mathrm{H}_{3}\right)$, there exists $M$ such that $\|x\| \neq M$. Let us set

$$
U=\{x \in C(I, \mathbb{R}):\|x\|<M\} .
$$

Note that the operator $\mathscr{F}: \bar{U} \rightarrow \mathscr{P}(C(I, \mathbb{R}))$ is a compact multivalued map and u.s.c. with convex closed values. From the choice of $U$, there is no $x \in \partial U$ such that $x \in \lambda \mathscr{F}(x)$ for some $\lambda \in(0,1)$. Consequently, by the nonlinear alternative of LeraySchauder type (Lemma 7), we deduce that $\mathscr{F}$ has a fixed point $x \in \bar{U}$ which is a solution of the problem (1).

\section{The lower semicontinuous case}

In the next result, $F$ is not necessarily convex valued. Our strategy to deal with this problem is based on the nonlinear alternative of Leray-Schauder type together with the selection theorem of Bressan and Colombo $^{24}$ for lower semicontinuous maps with decomposable values.

Let $X$ be a nonempty closed subset of a Banach space $E$ and $G: X \rightarrow \mathscr{P}(E)$ be a multivalued operator with nonempty closed values. $G$ is lower semicontinuous if the set $\{y \in X: G(y) \cap B \neq \varnothing\}$ is open for any open set $B$ in $E$. Let $A$ be a subset of $[0, T] \times \mathbb{R}$. $A$ is $\mathscr{L} \otimes \mathscr{B}$ measurable if $A$ belongs to the $\sigma$-algebra generated by all sets of the form $\mathscr{J} \times \mathscr{D}$, where $\mathscr{J}$ is Lebesgue measurable in $[0, T]$ and $\mathscr{D}$ is Borel measurable in $\mathbb{R}$. A subset $\mathscr{A}$ of $L^{1}([0, T], \mathbb{R})$ is decomposable if for all $u, v \in \mathscr{A}$ and measurable $\mathscr{J} \subset[0, T]=J$, the function $u \chi_{\mathscr{J}}+v \chi_{J-\mathscr{g}} \in \mathscr{A}$, where $\chi_{\mathscr{g}}$ stands for the characteristic function of $\mathscr{J}$.

Let $F:[0, T] \times \mathbb{R} \rightarrow \mathscr{P}(\mathbb{R})$ be a multivalued map with nonempty compact values. Define a multivalued operator $\mathscr{F}: C([0, T] \times \mathbb{R}) \rightarrow \mathscr{P}\left(L^{1}([0, T], \mathbb{R})\right)$ associated with $F$ by

$$
\begin{array}{r}
\mathscr{F}(x)=\left\{w \in L^{1}([0, T], \mathbb{R}): w(t) \in F(t, x(t))\right. \\
\text { for almost all } t \in[0, T]\},
\end{array}
$$

which is called the Nemytskii operator associated with $F$.

Let $F:[0, T] \times \mathbb{R} \rightarrow \mathscr{P}(\mathbb{R})$ be a multivalued function with nonempty compact values. We say $F$ is of lower semicontinuous type if its associated Nemytskii operator $\mathscr{F}$ is lower semicontinuous and has nonempty closed and decomposable values.

Lemma 8 (Ref. 25) Let $Y$ be a separable metric space and let $N: Y \rightarrow \mathscr{P}\left(L^{1}([0, T], \mathbb{R})\right)$ be a multivalued operator and is lower semicontinuous and has nonempty closed and decomposable values. Then $N$ has a continuous selection, i.e., there exists a continuous (single-valued) function $g: Y \rightarrow L^{1}([0, T], \mathbb{R})$ such that $g(x) \in N(x)$ for every $x \in Y$.

Theorem 2 Assume that $\left(\mathrm{H}_{2}\right),\left(\mathrm{H}_{3}\right)$, and the following condition holds.

$\left(\mathrm{H}_{4}\right) \quad F:[0, T] \times \mathbb{R} \rightarrow \mathscr{P}(\mathbb{R})$ is a nonempty compactvalued multivalued map such that

(i) $(t, x) \mapsto F(t, x)$ is $\mathscr{L} \otimes \mathscr{B}$ measurable;

(ii) $x \mapsto F(t, x)$ is lower semicontinuous for each $t \in[0, T]$.

Then the boundary value problem (1) has at least one solution on $[0, T]$. 
Proof: It follows from $\left(\mathrm{H}_{2}\right)$ and $\left(\mathrm{H}_{4}\right)$ that $F$ is of lower semicontinuous type. Then from Lemma 8 there exists a continuous function $f$ : $C^{2}([0, T], \mathbb{R}) \rightarrow L^{1}([0, T], \mathbb{R})$ such that $f(x) \in \mathscr{F}(x)$ for all $x \in C([0, T], \mathbb{R})$.

Consider the problem

$$
\left.\begin{array}{l}
D^{q} x(t)=f(x(t)), 0<t<T, \\
x(0)=0, \quad \alpha x(T)=\sum_{i=1}^{m} \beta_{i} I_{\eta_{i}}^{\gamma_{i}, \delta_{i}} x\left(\xi_{i}\right) .
\end{array}\right\}
$$

Observe that if $x \in C^{2}([0, T], \mathbb{R})$ is a solution of (8), then $x$ is a solution to (1). To transform (8) into a fixed point problem, we define the operator $\mathscr{F}$ by

$$
\begin{aligned}
\overline{\mathscr{F}} x(t)=J^{q} f(x(t))- & \frac{t^{q-1}}{\Lambda}\left(\alpha J^{q} f(x(T))\right. \\
& \left.-\sum_{i=1}^{m} \beta_{i} i_{\eta_{i}}^{\gamma_{i}, \delta_{i}} J^{q} f\left(\left(\xi_{i}\right)\right)\right) .
\end{aligned}
$$

It can easily be shown that $\overline{\mathscr{F}}$ is continuous and completely continuous. The remaining part of the proof is similar to that of Theorem 1 .

\section{The Lipschitz case}

In this subsection we prove the existence of solutions to (1) with a not necessarily nonconvex-valued right-hand side by applying a fixed-point theorem for multivalued maps due to Covitz and Nadler ${ }^{26}$.

Let $(X, d)$ be a metric space induced from the normed space $(X ;\|\cdot\|)$. Consider $H_{d}: \mathscr{P}(X) \times$ $\mathscr{P}(X) \rightarrow \mathbb{R} \cup\{\infty\}$ given by

$$
H_{d}(A, B)=\max \left\{\sup _{a \in A} d(a, B), \sup _{b \in B} d(A, b)\right\},
$$

where $d(A, b)=\inf _{a \in A} d(a ; b)$ and $d(a, B)=$ $\inf _{b \in B} d(a ; b)$. Then $\left(\mathscr{P}_{\mathrm{cl}, \mathrm{b}}(X), H_{d}\right)$ is a metric space $^{27}$.

A multivalued operator $N: X \rightarrow \mathscr{P}_{\mathrm{cl}}(X)$ is called

(i) $\gamma$-Lipschitz if and only if there exists $\gamma>0$ such that $H_{d}(N(x), N(y)) \leqslant \gamma d(x, y)$ for each $x, y \in$ $X$;

(ii) a contraction if and only if it is $\gamma$-Lipschitz with $\gamma<1$.

Lemma 9 (Ref. 26) Let $(X, d)$ be a complete metric space. If $N: X \rightarrow \mathscr{P}_{\mathrm{cl}}(X)$ is a contraction then Fix $N \neq$ $\varnothing$.

Theorem 3 Assume that

$\left(\mathrm{A}_{1}\right) F:[0, T] \times \mathbb{R} \rightarrow \mathscr{P}_{\mathrm{cp}}(\mathbb{R})$ is such that $F(\cdot, x)$ : $[0, T] \rightarrow \mathscr{P}_{\mathrm{cp}}(\mathbb{R})$ is measurable for each $x \in \mathbb{R}$;
$\left(\mathrm{A}_{2}\right) H_{d}(F(t, x), F(t, \bar{x})) \leqslant m(t)|x-\bar{x}|$ for almost all $t \in[0, T]$ and $x, \bar{x} \in \mathbb{R}$ with $m \in C\left([0, T], \mathbb{R}^{+}\right)$ and $d(0, F(t, 0)) \leqslant m(t)$ for almost all $t \in[0, T]$.

Then the boundary value problem (1) has at least one solution on $[0, T]$ if $\|m\| \Psi<1$, i.e.,

$$
\begin{aligned}
& \|m\|\left\{\frac{T^{q}}{\Gamma(q+1)}+\frac{|\alpha| T^{2 q-1}}{|\Lambda| \Gamma(q+1)}\right. \\
& \left.\quad+\frac{T^{q-1}}{|\Lambda| \Gamma(q+1)} \sum_{i=1}^{m} \frac{\left|\beta_{i}\right| \xi_{i}^{q} \Gamma\left(\gamma_{i}+q / \eta_{i}+1\right)}{\Gamma\left(\gamma_{i}+q / \eta_{i}+\delta_{i}+1\right)}\right\}<1 .
\end{aligned}
$$

Proof: Consider the operator $\mathscr{F}$ defined by (7). Observe that the set $S_{F, x}$ is nonempty for each $x \in$ $C([0, T], \mathbb{R})$ by the assumption $\left(\mathrm{A}_{1}\right)$. Thus $F$ has a measurable selection (see Theorem III.6 of Ref. 28). Now we show that the operator $\mathscr{F}$ is closed and a contraction. We show that $\mathscr{F}(x) \in \mathscr{P}_{\mathrm{cl}}((C[0, T], \mathbb{R}))$ for each $x \in C([0, T], \mathbb{R})$. Let $\left\{u_{n}\right\}_{n \geqslant 0} \in \mathscr{F}(x)$ be such that $u_{n} \rightarrow u(n \rightarrow \infty)$ in $C([0, T], \mathbb{R})$. Then $u \in C([0, T], \mathbb{R})$ and there exists $v_{n} \in S_{F, x_{n}}$ such that, for each $t \in[0, T]$,

$$
\begin{aligned}
u_{n}(t)=J^{q} v_{n}(s)(t)- & \frac{t^{q-1}}{\Lambda}\left(\alpha J^{q} v_{n}(s)(T)\right. \\
& \left.-\sum_{i=1}^{m} \beta_{i} I_{\eta_{i}}^{\gamma_{i}, \delta_{i}} J^{q} v_{n}(s)\left(\xi_{i}\right)\right) .
\end{aligned}
$$

As $F$ has compact values, it follows that the sequence $v_{n}$ converges to $v$ in $L^{1}([0, T], \mathbb{R})$. Thus $v \in S_{F, x}$ and for each $t \in[0, T]$ we have

$$
\begin{array}{r}
u_{n}(t) \rightarrow v(t)=J^{q} v(s)(t)-\frac{t^{q-1}}{\Lambda}\left(\alpha J^{q} v(s)(T)\right. \\
\left.-\sum_{i=1}^{m} \beta_{i} I_{\eta_{i}}^{\gamma_{i}, \delta_{i}} J^{q} v(s)\left(\xi_{i}\right)\right) .
\end{array}
$$

Hence $u \in \mathscr{F}(x)$.

Next we show that there exists $\delta<1$ ( $\delta=$ $\|m\| \Psi$ ) such that

$$
H_{d}(\mathscr{F}(x), \mathscr{F}(\bar{x})) \leqslant \delta\|x-\bar{x}\|
$$

for each $x, \bar{x} \in C^{2}([0, T], \mathbb{R}) . \quad$ Let $x, \bar{x} \in$ $C^{2}([0, T], \mathbb{R})$ and $h_{1} \in \mathscr{F}(x)$. Then there exists $v_{1}(t) \in F(t, x(t))$ such that, for each $t \in[0, T]$,

$$
\begin{aligned}
h_{1}(t)=J^{q} v_{1}(s)(t)- & \frac{t^{q-1}}{\Lambda}\left(\alpha J^{q} v_{1}(s)(T)\right. \\
& \left.-\sum_{i=1}^{m} \beta_{i} I_{\eta_{i}}^{\gamma_{i}, \delta_{i}} J^{q} v_{1}(s)\left(\xi_{i}\right)\right) .
\end{aligned}
$$


By $\left(\mathrm{A}_{2}\right)$ we have

$$
H_{d}(F(t, x), F(t, \bar{x})) \leqslant m(t)|x(t)-\bar{x}(t)| .
$$

Hence there exists $w \in F(t, \bar{x}(t))$ such that

$$
\left|v_{1}(t)-w(t)\right| \leqslant m(t)|x(t)-\bar{x}(t)|, \quad t \in[0, T] .
$$

Define $U:[0, T] \rightarrow \mathscr{P}(\mathbb{R})$ by

$$
U(t)=\left\{w \in \mathbb{R}:\left|v_{1}(t)-w\right| \leqslant m(t)|x(t)-\bar{x}(t)|\right\} .
$$

Since the multivalued operator $U(t) \cap F(t, \bar{x}(t))$ is measurable (Proposition III.4 of Ref. 28), there exists a function $v_{2}(t)$ which is a measurable selection for $U$. Thus $v_{2}(t) \in F(t, \bar{x}(t))$ and for each $t \in[0, T]$ we have $\left|v_{1}(t)-v_{2}(t)\right| \leqslant m(t)|x(t)-\bar{x}(t)|$. For each $t \in[0, T]$, let us define

$$
\begin{aligned}
h_{2}(t)=J^{q} v_{2}(s)(t)- & \frac{t^{q-1}}{\Lambda}\left(\alpha J^{q} v_{2}(s)(T)\right. \\
& \left.-\sum_{i=1}^{m} \beta_{i} I_{\eta_{i}}^{\gamma_{i}, \delta_{i}} J^{q} v_{2}(s)\left(\xi_{i}\right)\right) .
\end{aligned}
$$

Thus

$$
\begin{aligned}
\mid h_{1}(t) & -h_{2}(t) \mid \\
\leqslant & J^{q}\left|v_{1}(s)-v_{2}(s)\right|(t)+\frac{t^{q-1}}{|\Lambda|}\left(\alpha J^{q} \mid v_{1}(s)\right. \\
& \left.-v_{2}(s)\left|(T)+\sum_{i=1}^{m} \beta_{i} I_{\eta_{i}}^{\gamma_{i}, \delta_{i}} J^{q}\right| v_{1}(s)-v_{2}(s) \mid\left(\xi_{i}\right)\right) \\
\leqslant & \|m\|\|x-\bar{x}\|\left(\frac{T^{q}}{\Gamma(q+1)}+\frac{|\alpha| T^{2 q-1}}{|\Lambda| \Gamma(q+1)}\right. \\
& \left.+\frac{T^{q-1}}{|\Lambda| \Gamma(q+1)} \sum_{i=1}^{m} \frac{\left|\beta_{i}\right| \xi_{i}^{q} \Gamma\left(\gamma_{i}+q / \eta_{i}+1\right)}{\Gamma\left(\gamma_{i}+q / \eta_{i}+\delta_{i}+1\right)}\right) .
\end{aligned}
$$

Hence

$$
\begin{aligned}
\| h_{1}- & h_{2} \| \\
& \leqslant\|m\|\|x-\bar{x}\|\left(\frac{T^{q}}{\Gamma(q+1)}+\frac{|\alpha| T^{2 q-1}}{|\Lambda| \Gamma(q+1)}\right. \\
& \left.+\frac{T^{q-1}}{|\Lambda| \Gamma(q+1)} \sum_{i=1}^{m} \frac{\left|\beta_{i}\right| \xi_{i}^{q} \Gamma\left(\gamma_{i}+q / \eta_{i}+1\right)}{\Gamma\left(\gamma_{i}+q / \eta_{i}+\delta_{i}+1\right)}\right) .
\end{aligned}
$$

Analogously, interchanging the roles of $x$ and $\bar{x}$,

$$
H_{d}(\mathscr{F}(x), \mathscr{F}(\bar{x})) \leqslant \delta\|x-\bar{x}\|
$$

with $\delta<1$ where

$$
\begin{aligned}
\delta= & \|m\|\left(\frac{T^{q}}{\Gamma(q+1)}+\frac{|\alpha| T^{2 q-1}}{|\Lambda| \Gamma(q+1)}\right. \\
& \left.+\frac{T^{q-1}}{|\Lambda| \Gamma(q+1)} \sum_{i=1}^{m} \frac{\left|\beta_{i}\right| \xi_{i}^{q} \Gamma\left(\gamma_{i}+q / \eta_{i}+1\right)}{\Gamma\left(\gamma_{i}+q / \eta_{i}+\delta_{i}+1\right)}\right) .
\end{aligned}
$$

Thus $\mathscr{F}$ is a contraction. Hence it follows by Lemma 9 that $\mathscr{F}$ has a fixed point $x$ which is a solution of (1).

\section{Examples}

We now illustrate our main theorems with the help of three examples. Consider the following nonlocal boundary value problems for fractional differential inclusions with Erdélyi-Kober fractional integral boundary conditions:

$$
\left.\begin{array}{rl}
D^{3 / 2} x(t) \in F(t, x(t)), t \in(0,2), x(0)=0, \\
\frac{2}{3} x(2)=\frac{3}{2} I_{\sqrt{2} / 3}^{5 / 4,1 / 2} x\left(\frac{1}{4}\right) \\
+\frac{\ln 2}{\sqrt{3}} I_{\sqrt{3} / 4}^{4 / 9,3 / 2} x\left(\frac{1}{2}\right)+\frac{5}{3} I_{\sqrt{5} / 6}^{7 / 2,5} x\left(\frac{3}{4}\right) \\
+\frac{3}{\sqrt{5}} I_{3 / 8}^{8 / 3,2 / 9} x\left(\frac{5}{4}\right)+\frac{e}{\pi} I_{4 / 7}^{3 / 8,6 / \sqrt{7}} x\left(\frac{3}{2}\right) \\
+\frac{\pi}{6} I_{7 / 9}^{13 / 11, \sqrt{2} / \sqrt{3}} x\left(\frac{7}{4}\right) .
\end{array}\right\}
$$

Here we have $q=\frac{3}{2}, m=6, T=2, \alpha=\frac{2}{3}, \beta_{1}=\frac{3}{2}$, $\beta_{2}=\ln 2 / \sqrt{3}, \beta_{3}=\frac{5}{3}, \beta_{4}=3 / \sqrt{5}, \beta_{5}=e / \pi, \beta_{6}=$ $\pi / 6, \eta_{1}=\sqrt{2} / 3, \eta_{2}=\sqrt{3} / 4, \eta_{3}=\sqrt{5} / 6, \eta_{4}=\frac{3}{8}$, $\eta_{5}=\frac{4}{7}, \eta_{6}=\frac{7}{9}, \gamma_{1}=\frac{5}{4}, \gamma_{2}=\frac{4}{9}, \gamma_{3}=\frac{7}{2}, \gamma_{4}=\frac{8}{3}, \gamma_{5}=$ $\frac{3}{8}, \gamma_{6}=\frac{13}{11}, \delta_{1}=\frac{1}{2}, \delta_{2}=\frac{3}{2}, \delta_{3}=\frac{5}{2}, \delta_{4}=\frac{2}{9}, \delta_{5}=$ $6 / \sqrt{7}, \delta_{6}=\sqrt{2} / \sqrt{3}, \xi_{1}=\frac{1}{4}, \xi_{2}=\frac{1}{2}, \xi_{3}=\frac{3}{4}, \xi_{4}=\frac{5}{4}$, $\xi_{5}=\frac{3}{2}$, and $\xi_{6}=\frac{7}{4}$. This gives $|\Lambda|=1.030325363$ and $\Psi=6.111985724$.

(a) Let $F:[0,2] \times \mathbb{R} \rightarrow \mathscr{P}(\mathbb{R})$ be a multivalued map given by

$$
\begin{aligned}
& x \rightarrow F(t, x) \\
& =\left[\frac{\sin ^{2} x+\mathrm{e}^{-|x|}}{10(2+\cos t)}, \frac{1}{t+3}\left(\frac{x^{2}}{5(1+|x|)}+3\right)\right] .
\end{aligned}
$$

For $f \in F$ we have

$$
\begin{aligned}
|f| & \leqslant \max \left(\frac{\sin ^{2} x+\mathrm{e}^{-|x|}}{10(2+\cos t)}, \frac{1}{t+3}\left(\frac{x^{2}}{5(1+|x|)}+3\right)\right) \\
& \leqslant \frac{1}{t+3}\left(\frac{|x|}{5}+3\right), \quad x \in \mathbb{R} .
\end{aligned}
$$

Thus for $x \in \mathbb{R}$,

$$
\begin{aligned}
\|F(t, x)\|_{\mathscr{P}} & :=\sup \{|y|: y \in F(t, x)\} \\
& \leqslant \frac{1}{t+3}\left(\frac{|x|}{5}+3\right)=p(t) \psi(|x|),
\end{aligned}
$$

with $p(t)=1 /(t+3), \psi(|x|)=(|x| / 5)+3$. Hence there exists a constant $M>10.31499084$ satisfying $\left(\mathrm{H}_{3}\right)$. Thus all the conditions of Theorem 1 are 
satisfied. Hence the problem (9) with $F(t, x)$ given by (10) has at least one solution on $[0,2]$.

(b) Let $F:[0,2] \times \mathbb{R} \rightarrow \mathscr{P}(\mathbb{R})$ be a multivalued map given by

$$
x \rightarrow F(t, x)=\left[\mathrm{e}^{-x^{4}}+\frac{t}{3}, \frac{|x|}{1+2|x|}+t+\frac{3}{2}\right] .
$$

For $f \in F$ we have

$$
\begin{aligned}
|f| & \leqslant \max \left(\mathrm{e}^{-x^{4}}+\frac{t}{3}, \frac{|x|}{1+2|x|}+t+\frac{3}{2}\right) \\
& \leqslant 2+t, \quad x \in \mathbb{R} .
\end{aligned}
$$

Here,

$$
\begin{aligned}
\|F(t, x)\|_{\mathscr{P}} & :=\sup \{|y|: y \in F(t, x)\} \\
& \leqslant(2+t)=p(t) \psi(\|x\|), \quad x \in \mathbb{R},
\end{aligned}
$$

with $p(t)=2+t, \psi(\|x\|)=1$. By computing directly, there exists a constant $M>24.44794290$ satisfying $\left(\mathrm{H}_{3}\right)$. Then, by Theorem 2 , the problem (9) with $F(t, x)$ given by (11) has at least one solution on $[0,2]$.

(c) Consider the multivalued map $F:[0,2] \times$ $\mathbb{R} \rightarrow \mathscr{P}(\mathbb{R})$ given by

$$
x \rightarrow F(t, x)=\left[0, \frac{1}{8+t^{2}}\left(\frac{x^{2}+2|x|}{2(1+|x|)}+1\right)\right] .
$$

Then we have

$$
H_{d}(F(t, x), F(t, \bar{x})) \leqslant \frac{1}{8+t^{2}}|x-\bar{x}| .
$$

Let $m(t)=1 /\left(8+t^{2}\right)$. Then $H_{d}(F(t, x), F(t, \bar{x})) \leqslant$ $m(t)|x-\bar{x}|$ with $d\left(0, F(t, 0) \leqslant m(t)\right.$ and $\|m\|=\frac{1}{8}$. We can show that

$$
\|m\| \Psi=0.7639982155<1 .
$$

Thus all the conditions of Theorem 3 are fulfilled. Hence by the conclusion of Theorem 3 , the problem (9) with $F(t, x)$ given by (12) has at least one solution on $[0,2]$.

\section{REFERENCES}

1. Heymans N, Bauwens JC (1994) Fractal rheological models and fractional differential equations for viscoelastic behavior. Rheol Acta 33, 210-9.

2. Metzler R, Klafter J (2000) The random walk's guide to anomalous diffusion: a fractional dynamics approach. Phys Rep 339, 1-77.

3. Arena P, Fortuna L, Porto D (2000) Chaotic behavior in noninteger-order cellular neural networks. Phys Rev E 61, 776-81.
4. Picozzi S, West BJ (2002) Fractional Langevin model of memory in financial markets. Phys Rev E 66, 046118.

5. Podlubny I (1999) Fractional Differential Equations, Academic Press, San Diego.

6. Kilbas AA, Srivastava HM, Trujillo JJ (2006) Theory and Applications of Fractional Differential Equations, North-Holland Mathematics Studies, 204. Elsevier, Amsterdam.

7. Agarwal RP, Zhou Y, He Y (2010) Existence of fractional neutral functional differential equations. Comput Math Appl 59, 1095-100.

8. Baleanu D, Mustafa OG, Agarwal RP (2011) On L ${ }^{p}$ solutions for a class of sequential fractional differential equations. Appl Math Comput 218, 2074-81.

9. Ahmad B, Nieto JJ (2011) Riemann-Liouville fractional integro-differential equations with fractional nonlocal integral boundary conditions. Bound Value Probl 2011, 36.

10. Ahmad B, Ntouyas SK (2014) A fully Hadamard-type integral boundary value problem of a coupled system of fractional differential equations. Fract Calc Appl Anal 17, 348-60.

11. Ahmad B, Ntouyas SK (2015) Nonlocal fractional boundary value problems with slit-strips integral boundary conditions. Fract Calc Appl Anal 18, 261-80.

12. Ahmad B, Ntouyas SK, Alsaedi A (2011) New existence results for nonlinear fractional differential equations with three-point integral boundary conditions. Adv Difference Equat 2011, 107384

13. Zhou Y, Peng L (2017) On the time-fractional NavierStokes equations. Comput Math Appl 73, 874-91.

14. Zhou Y, Peng L (2017) Weak solution of the timefractional Navier-Stokes equations and optimal control. Comput Math Appl 73, 1016-27.

15. Kober H (1940) On fractional integrals and derivatives. Q J Math 11, 193-211.

16. Kalla SL, Kiryakova VS (1990) An $H$-function generalized fractional calculus based upon compositions of Erdélyi-Kober operators in $L_{p}$. Math Jpn 35, 1-21.

17. Kiryakova VS (1994) Generalized Fractional Calculus and Applications, Pitman Research Notes in Mathematics 301, Longman, Harlow.

18. Samko SG, Kilbas AA, Marichev OI (1993) Fractional Integrals and Derivatives: Theory and Applications, Gordon and Breach, New York.

19. Thongsalee N, Ntouyas SK, Tariboon J (2016) Nonlinear Riemann-Liouville fractional differential equations with nonlocal Erdélyi-Kober fractional integral conditions. Fract Calc Appl Anal 19, 480-97.

20. Deimling K (1992) Multivalued Differential Equations, Walter De Gruyter, Berlin.

21. Hu Sh Papageorgiou N (1997) Handbook of Multivalued Analysis, Theory I, Kluwer, Dordrecht.

22. Lasota A, Opial Z (1965) An application of the Kakutani-Ky Fan theorem in the theory of ordinary 
differential equations. Bull Acad Polon Sci Sér Sci Math Astron Phys 13, 781-6.

23. Granas A, Dugundji J (2005) Fixed Point Theory, Springer-Verlag, New York.

24. Bressan A, Colombo G (1988) Extensions and selections of maps with decomposable values. Stud Math 90, 69-86.

25. Frigon M (1995) Théorèmes d'existence de solutions d'inclusions différentielles. In: Granas A, Frigon M (eds) Topological Methods in Differential Equations and Inclusions, NATO ASI Series C, Vol. 472, Kluwer, Dordrecht, pp 51-87.

26. Covitz H, Nadler Jr SB (1970) Multivalued contraction mappings in generalized metric spaces. Isr $J$ Math 8, 5-11.

27. Kisielewicz M (1991) Differential Inclusions and Optimal Control, Kluwer, Dordrecht, The Netherlands.

28. Castaing C, Valadier M (1977) Convex Analysis and Measurable Multifunctions, Lecture Notes in Mathematics 580, Springer-Verlag, Berlin. 